\title{
Metabolic Syndrome and Its Components Are Associated With Hypoxemia After Surgery for Acute Type A Aortic Dissection: an Observational Study
}

\author{
Like Zhang \\ Hebei Medical University First Affiliated Hospital \\ Lei Zhang \\ Hebei Medical University First Affiliated Hospital \\ Zengren Zhao ( $\square$ zzr-doctor@163.com ) \\ Hebei Medical University First Affiliated Hospital \\ Yun Liu \\ Hebei Medical University First Affiliated Hospital \\ Juzeng Wang \\ Hebei Medical University First Affiliated Hospital \\ Mengye Niu \\ Hebei Medical University First Affiliated Hospital \\ Xiansheng Zhao \\ Hebei Medical University First Affiliated Hospital
}

\section{Research article}

Keywords: Acute type A aortic dissection, metabolic syndrome, hypoxemia, components, scoring system

Posted Date: September 21st, 2021

DOI: https://doi.org/10.21203/rs.3.rs-900428/v1

License: (9) This work is licensed under a Creative Commons Attribution 4.0 International License. Read Full License 


\section{Abstract}

Background: The aim of this study was to explore whether or to what extent metabolic syndrome (METs) and its components were associated with hypoxemia in acute type $A$ aortic dissection (ATAAD) patients after surgery.

Methods: This study involved 271 inpatients who underwent surgery. Demographic and clinical data were collected. Subgroup analysis, mixed model regression analysis, and receiver operating characteristic (ROC) curve analysis were performed, and a scoring system was evaluated.

Results: The 271 inpatients were assigned to the hypoxemia group $(n=48)$ or no hypoxemia group $(n=223)$ regardless of METs status. Compared to the no hypoxemia group, the hypoxemia group had a higher incidence of METs. Hypoxemia was present in $0 \%, 3.7 \%, 19.8 \%$, $51.5 \%, 90.0 \%$ and $100 \%$ in the groups of individuals who met the diagnostic criteria of MetS $0,1,2,3,4$ and 5 times, respectively. In the multivariable logistic regression analysis, BMI quartile was still a risk factor for hypoxemia after adjustment for other risk factors. After adjustment for potential confounding factors, METs was an independent risk factor for hypoxemia in several models. After assigning a score for each METs component present, the AUCs were 0.852 (95\% Cl: $0.789-0.914)$ in all patients, 0.728 (95\% Cl: $0.573-0.882)$ in patients with METs and 0.744 (95\% Cl: $0.636-0.853)$ in patients without METs according to receiver operating characteristic analysis.

Conclusions: METs, especially body mass index, confers a greater risk of hypoxemia in ATAAD after surgery.

\section{Background}

Acute type A aortic dissection (ATAAD) is a life-threatening cardiovascular disease with high mortality; the mortality rate is approximately $27 \%$ after surgery and approximately $58 \%$ with noninvasive treatment [1]. Despite significant improvements in surgical techniques, postoperative mortality is still high for ATAAD due to the incidence of complications [2]. Hypoxemia is the most common symptom of acute lung injury and is characterized by a ratio of arterial partial pressure of oxygen to fraction of inspired oxygen (PaO2/FiO2) $\leq 300 \mathrm{mmHg}$, which also leads to increased mortality [3]. The underlying mechanisms of hypoxemia in ATAAD remain elusive. Previous studies found that systolic blood pressure levels, body mass index (BMI), and obesity were important indicators of the prognosis of hypoxemia in ATAAD [4-6]. In addition, glucose and hyperlipidemia are associated with hypoxemia in other systems [7-8]. Metabolic syndrome (METs) is characterized by a cluster of risk components, including abdominal obesity, hyperglycemia, dyslipidemia and hypertension [9]. The relationship between hypoxemia and METs has not been described in ATAAD. In the present study, we investigated the association of METs and its components with the incidence of hypoxemia and determined the usefulness of METs for diagnosis of and risk assessment in ATAAD in clinical practice, providing new insight into the incidence of hypoxemia.

\section{Methods}

\section{Study cohort}

This is an observational and retrospective study. A total of 271 consecutive ATAAD patients who received treatment in the Department of General Surgery at the First Hospital of Hebei Medical University were enrolled in this study from January 2015 to January 2021. The inclusion criteria were as follows: 1) diagnosed with ATAAD confirmed by CT angiography of the aorta and 2) underwent surgical treatment. The major exclusion criteria included the following: 1) patients with respiratory system diseases; 2) patients who did not undergo surgery; and 3) patients who suffered from any perioperative complications. According to arterial blood gas analysis, patients with PaO2/FiO2 $\leq 300 \mathrm{mmHg}$ for the first 2 days after the operation were included in the hypoxemia group. Patients with PaO2/FiO2 greater than $300 \mathrm{mmHg}$ formed the no hypoxemia group. The study was approved by the Institutional Review Board of the First Hospital of Hebei Medical University. All subjects provided written informed consent. The detailed recruitment process is shown in Figure 1.

\section{Metabolic syndrome}

According to the criteria of the American National Cholesterol Education Program [10], MetS was defined as the presence of three or more of the following criteria: body mass index (BMI) $>30 \mathrm{~kg} / \mathrm{m}^{2}$, high-density lipoprotein (HDL) $<50 \mathrm{mg} / \mathrm{dL}$ among women and $<40 \mathrm{mg} / \mathrm{dL}$ among men, fasting plasma triglycerides (TG) $\geq 150 \mathrm{mg} / \mathrm{dL}$, systolic blood pressure (SBP) $\geq 130 \mathrm{mmHg}$, diastolic blood pressure (DBP) $\geq 85 \mathrm{mmHg}$, fasting plasma glucose (FPG) $\geq 100 \mathrm{mg} / \mathrm{dL}$ or previously diagnosed type 2 diabetes mellitus (T2DM).

\section{Baseline demographic and clinical characteristics}

Data regarding sex, age, body mass index (BMI), blood pressure, heart rate (HR), systolic blood pressure (SBP), diastolic blood pressure (DBP), ventricular ejection fraction (LVEF), history of hypertension (HT), type 2 diabetes mellitus (T2DM), coronary artery disease (CAD) and thoracic surgery were collected. Preoperative laboratory tests were performed within $24 \mathrm{~h}$ before surgery, including tests for fasting blood glucose (FBG), triglycerides (TG), high-density lipoprotein cholesterol (HDL-C), white blood cells (WBCs), platelets (PLTs), creatinine (Cr), uric acid (UA), 
troponin I, and red blood cells (RBCs). Surgery variables included length of surgery, cardiopulmonary bypass time, cross-clamp time, circulatory arrest, minimum temperature, ICU stay time, hospital stay time, $\mathrm{PaO} 2 / \mathrm{FiO} 2$ and mechanical ventilation time.

\section{Statistical methods}

Statistical computations were performed using SPSS v24.0 (IBM Inc., Armonk, NY, USA). Continuous variables are reported as the mean \pm standard deviation for normally distributed data or as the median and quartiles (Q1, Q3) for nonnormally distributed data. Discrete variables are expressed as frequencies and percentages and were compared using the chi-square test. Multivariable logistic regression analyses were performed to detect the relationship between hypoxemia and METs. In the multivariate analysis, odds ratios (ORs) and 95\% confidence intervals (Cls) for hypoxemia were calculated using a logistic regression model after adjusting for potential confounding variables. To verify the robustness of our results, subgroup analyses were performed to explore the association between the number of MetS components and hypoxemia. These predictors of metabolic syndrome components were assigned points based on their regression coefficient, and a scoring system was produced. Receiver operating characteristic (ROC) curves were constructed, and the areas under the curves (AUCs) were calculated to assess the discriminatory power of the scoring system for MetS. A two-sided $p$ value $<0.05$ was considered statistically significant.

\section{Results}

\section{Baseline demographic and clinical characteristics}

Table 1 summarizes the clinical characteristics of the hypoxemia group $(n=48)$ and no hypoxemia group $(n=223)$. The mean age was $53.4 \pm$ 7.2 and $52.9 \pm 6.2$ years in the two groups, respectively. The incidence of aortic valve disease, Marfan syndrome, CAD, and history of thoracic surgery were not significantly different between the groups (all $p>0.05$ ). Compared to the control group, the hypoxemia group had significantly greater BMI, SBP, TG and WBC values and a longer length of surgery, ICU stay time, hospital stay time and mechanical ventilation time, and the differences were statistically significant (all $P<0.05$ ). Similarly, there were statistically significant differences in the incidence of HT, T2DM, metabolic syndrome and smoking (all $p>0.05$ ). 
Table 1

clinical characteristics in two group.

\begin{tabular}{|c|c|c|c|c|}
\hline \multirow[t]{2}{*}{ Variables } & \multirow{2}{*}{$\begin{array}{l}\text { Hypoxemia group } \\
n=48\end{array}$} & \multirow{2}{*}{$\begin{array}{l}\text { No Hypoxemia group } \\
n=223\end{array}$} & \multirow[t]{2}{*}{$x^{2} / t$} & \multirow[t]{2}{*}{$P$ Value } \\
\hline & & & & \\
\hline Male,n(\%) & $37(77.1)$ & 164(73.5) & 0.258 & 0.611 \\
\hline Age,years & $53.4 \pm 7.2$ & $52.9 \pm 6.2$ & 0.555 & 0.579 \\
\hline $\mathrm{BMI}, \mathrm{Kg} / \mathrm{m}^{2}$ & $29.2 \pm 4.1$ & $25.8 \pm 2.7$ & -7.277 & $<0.001$ \\
\hline$H T, n(\%)$ & $32(66.7)$ & $113(50.7)$ & 4.062 & 0.044 \\
\hline T2DM,n(\%) & 19(39.6) & $26(11.7)$ & 22.241 & $<0.001$ \\
\hline Aortic valve disease,n(\%) & $4(8.3)$ & $16(7.2)$ & 0.078 & 0.781 \\
\hline Metabolic syndrome,n(\%) & $27(56.3)$ & $17(7.6)$ & 68.673 & $<0.001$ \\
\hline smoker,n(\%) & $31(64.6)$ & $96(43.0)$ & 7.355 & 0.007 \\
\hline Marfan syndrome,n(\%) & $1(2.1)$ & $1(0.4)$ & 1.441 & 0.230 \\
\hline CAD,n(\%) & $7(14.6)$ & $34(15.2)$ & 0.014 & 0.907 \\
\hline History of thoracic surgery,n(\%) & $2(4.2)$ & $21(9.4)$ & 1.402 & 0.236 \\
\hline SBP,mmHg & $150.5 \pm 13.4$ & $143.4 \pm 13.8$ & -3.264 & 0.001 \\
\hline $\mathrm{DBP}, \mathrm{mmHg}$ & $80.3 \pm 6.2$ & $79.1 \pm 6.7$ & -1.110 & 0.268 \\
\hline $\mathrm{HR}, \mathrm{bpm}$ & $70.1 \pm 12.3$ & $71.7 \pm 11.1$ & 0.177 & 0.383 \\
\hline LVEF,\% & $56.9 \pm 6.0$ & $57.4 \pm 6.5$ & 0.480 & 0.632 \\
\hline Troponin I, ng/mL & $0.01(0,0.01)$ & $0(0,0.01)$ & -1.103 & 0.270 \\
\hline $\mathrm{FBG}, \mathrm{mmol} / \mathrm{L}$ & $5.0 \pm 0.7$ & $4.9 \pm 0.4$ & -1.229 & 0.220 \\
\hline TG, mmol/L & $1.5(1.1,2.6)$ & $1.2(1.0,1.5)$ & -2.620 & 0.009 \\
\hline $\mathrm{HDL}-\mathrm{C}, \mathrm{mmol} / \mathrm{L}$ & $1.2(0.9,1.8)$ & 1.6(1.1,2.2) & -3.925 & $<0.001$ \\
\hline $\mathrm{WBC}, 10^{12} / \mathrm{L}$ & $11.4 \pm 1.9$ & $10.0 \pm 1.4$ & -5.961 & $<0.001$ \\
\hline $\mathrm{PLT}, 10^{9} / \mathrm{L}$ & $235.5 \pm 67.8$ & $217.6 \pm 58.9$ & -1.653 & 0.100 \\
\hline $\mathrm{RBC}, 10^{12} / \mathrm{L}$ & $4.6 \pm 0.6$ & $4.6 \pm 0.5$ & -0.426 & 0.670 \\
\hline $\mathrm{Cr}, \mu \mathrm{mol} / \mathrm{L}$ & $75.4 \pm 16.4$ & $76.2 \pm 21.3$ & 0.246 & 0.806 \\
\hline $\mathrm{UA}, \mu \mathrm{mol} / \mathrm{L}$ & $349.0 \pm 91.9$ & $338.6 \pm 76.9$ & 0.725 & 0.469 \\
\hline eGFR, mL/(min·1.73 m2) & $90.9 \pm 14.3$ & $90.5 \pm 16.2$ & -0.145 & 0.885 \\
\hline Length of surgery, min & $287.3 \pm 19.4$ & $276.2 \pm 24.9$ & -2.913 & 0.004 \\
\hline Cardiopulmonary bypass time,min & $167.5 \pm 22.5$ & $170.0 \pm 23.0$ & 0.674 & 0.501 \\
\hline Cross-clamp time,min & $87.7 \pm 13.6$ & $88.7 \pm 13.0$ & 0.465 & 0.642 \\
\hline Circulatory arrest,min & $43.4 \pm 7.3$ & $42.1 \pm 7.5$ & -1.076 & 0.283 \\
\hline Minimum temperature, ${ }^{\circ} \mathrm{C}$ & $26.0 \pm 0.5$ & $26.0 \pm 0.5$ & 1.105 & 0.270 \\
\hline ICU stay time,day & $6.7 \pm 1.6$ & $5.4 \pm 1.5$ & -5.488 & $<0.001$ \\
\hline Hospital stay time,day & $20.0 \pm 3.7$ & $17.1 \pm 4.2$ & -4.522 & $<0.001$ \\
\hline $\mathrm{PaO} 2 / \mathrm{FiO} 2, \mathrm{mmHg}$ & $256.4 \pm 24.4$ & $330.8 \pm 13.5$ & 29.346 & $<0.001$ \\
\hline
\end{tabular}

BMI: body mass index; HT: Hypertension; T2DM: type 2 diabetes mellitus; SBP: systolic blood pressure; DBP: diastolic blood pressure; HR: heart rate; FBG: Fasting blood glucose; TG: Triglycerides; HDL-C: high-density lipoprotein cholesterol; WBC: white blood cell; PLT: Platelet; Cr: creatinine; UA: Uric acid; LVEF: ventricular ejection fraction. 


\begin{tabular}{|c|c|c|c|c|}
\hline \multirow[t]{2}{*}{ Variables } & \multirow{2}{*}{$\begin{array}{l}\text { Hypoxemia group } \\
n=48\end{array}$} & \multirow{2}{*}{$\begin{array}{l}\text { No Hypoxemia group } \\
n=223\end{array}$} & \multirow[t]{2}{*}{$x^{2} / t$} & \multirow[t]{2}{*}{$P$ Value } \\
\hline & & & & \\
\hline Mechanical ventilation time,hour & $39.2 \pm 21.9$ & $21.1 \pm 8.2$ & -9.587 & $<0.001$ \\
\hline Elevated BMI,n (\%) & 18(37.5) & $11(4.9)$ & 43.839 & $<0.001$ \\
\hline Elevated BP,n (\%) & $47(97.9)$ & 203(91.0) & 2.619 & 0.106 \\
\hline Elevated FBG,n (\%) & 23(47.9) & $31(13.9)$ & 28.643 & $<0.001$ \\
\hline Reduced HDL-C,n (\%) & $25(52.1)$ & $42(18.8)$ & 23.462 & $<0.001$ \\
\hline Elevated TG,n (\%) & 16(33.3) & 26(11.7) & 14.168 & $<0.001$ \\
\hline
\end{tabular}

\section{METs incidence and clinical characteristics}

Participants were divided into six groups according to whether they met $0,1,2,3,4$ or 5 of the METs diagnostic criteria, and there were 10 (3.7\%), $136(50.2 \%), 81(29.9 \%), 33(12.2 \%), 10(3.7 \%)$ and $1(0.3 \%)$ individuals in the respective groups. Hypoxemia was present in $0 \%, 3.7 \%$, $19.8 \%, 51.5 \%, 90.0 \%$ and $100 \%$ of the six groups, with significant differences among groups $(P<0.05$, Table 2 Fig. 2$)$. The prevalence of males was the highest in the 0 group. There were significant differences among the six groups in terms of mechanical ventilation time, WBC, $\mathrm{PaO} 2 / \mathrm{FiO} 2$, cross-clamp time and LVEF (all $P<0.001$ ). The MetS components $\mathrm{BMI}, \mathrm{HT}, \mathrm{T} 2 \mathrm{DM}, \mathrm{SBP}$, and TG increased with increasing numbers of traits. For comparisons among groups, the greatest difference was in BMI. $(P<0001$, Table 2$)$. 
Table 2

Baseline characteristics of the number of METs.

\begin{tabular}{|c|c|c|c|c|c|c|c|c|}
\hline \multirow[t]{3}{*}{ Variables } & \multicolumn{8}{|c|}{ the number of the presence of MetS } \\
\hline & \multirow{2}{*}{$\begin{array}{l}0 \\
n=10\end{array}$} & \multirow{2}{*}{$\begin{array}{l}1 \\
n=136\end{array}$} & \multirow{2}{*}{$\begin{array}{l}2 \\
n=81\end{array}$} & \multirow{2}{*}{$\begin{array}{l}3 \\
n=33\end{array}$} & \multirow{2}{*}{$\begin{array}{l}4 \\
n=10\end{array}$} & \multirow{2}{*}{$\begin{array}{l}5 \\
n=1\end{array}$} & \multirow[t]{2}{*}{$P$ Value } & \multirow[t]{2}{*}{$P \otimes 0.05$} \\
\hline & & & & & & & & \\
\hline Male,n(\%) & $8(80.0)$ & $101(74.3)$ & $62(76.5)$ & $21(63.6)$ & $8(80.0)$ & $1(100.0)$ & 0.723 & $c, e, i, l$ \\
\hline Age,years & $52.1 \pm 3.9$ & $53.1 \pm 6.5$ & $53.0 \pm 5.6$ & $52.3 \pm 7.7$ & $52.9 \pm 7.4$ & 65 & 0.521 & e,l \\
\hline $\mathrm{BMI}, \mathrm{Kg} / \mathrm{m} 2$ & $24.3 \pm 1.7$ & $25.4 \pm 2.3$ & $26.9 \pm 2.9$ & $27.2 \pm 3.4$ & $33.7 \pm 5.0$ & 35.0 & $\varangle 0.001$ & b,c,d,e,f,g,h,i,k,l,m,n \\
\hline $\mathrm{HT}, \mathrm{n}(\%)$ & $0(0)$ & $71(52.5)$ & $45(55.6)$ & $23(69.7)$ & $5(50.0)$ & $1(100.0)$ & 0.006 & a,b,c,d,e,i,l,n,o \\
\hline T2DM,n(\%) & $0(0)$ & $3(2.2)$ & 18(22.2) & 18(54.5) & $5(50.0)$ & $1(100.0)$ & $\varangle 0.001$ & b,c,d,e,g,h,i,l,m,n,o \\
\hline Hypoxemia,n(\%) & $0(0)$ & $5(3.7)$ & $16(19.8)$ & $17(51.5)$ & $9(90.0)$ & $1(100.0)$ & $\nabla 0.001$ & b,c,d,e,g,h,i,j,k,l, m,n,o \\
\hline SBP,mmHg & $123.8 \pm 6.5$ & $144.8 \pm 14.8$ & $144.7 \pm 11.7$ & $148.9 \pm 13.0$ & $149.7 \pm 9.4$ & 149 & $\nabla 0.001$ & $a, b, c, d, e$ \\
\hline DBP,mmHg & $72.2 \pm 6.7$ & $79.5 \pm 6.4$ & $79.3 \pm 6.8$ & $80.4 \pm 6.5$ & $81.5 \pm 6.6$ & 80 & 0.019 & $a, b, c, d$ \\
\hline HR,bpm & $68.8 \pm 5.3$ & $73.3 \pm 12.9$ & $70.5 \pm 9.3$ & $69.0 \pm 7.9$ & $65.2 \pm 12.9$ & 59 & 0.063 & \\
\hline $\mathrm{FBG}, \mathrm{mmol} / \mathrm{L}$ & $5.2 \pm 0.3$ & $4.9 \pm 0.4$ & $5.0 \pm 0.5$ & $5.1 \pm 0.6$ & $5.1 \pm 0.8$ & 6.3 & 0.002 & $a, e, f, g, i, l, n$ \\
\hline TG,mmol/L & $1.2(0.9,1.3)$ & $1.2(1.0,1.4)$ & $1.3(1.1,1.6)$ & $1.5(1.2,2.1)$ & $2.7(2.1,3.3)$ & 2.4 & $\bowtie 0.001$ & $c, d, e, f, g, h, i, k, m$ \\
\hline $\begin{array}{l}\text { Mechanical } \\
\text { ventilation } \\
\text { time,hour }\end{array}$ & $19.9 \pm 7.9$ & $21.6 \pm 9.2$ & $25.2 \pm 13.4$ & $29.9 \pm 12.8$ & $40.1 \pm 18.8$ & 36 & $\varangle 0.001$ & $d, f, g, h, k$ \\
\hline WBC,1012/L & $9.6 \pm 2.0$ & $10.1 \pm 1.4$ & $10.0 \pm 1.7$ & $10.6 \pm 1.5$ & $11.6 \pm 2.2$ & 10.3 & 0.024 & $\mathrm{~d}, \mathrm{~h}, \mathrm{k}$ \\
\hline PLT,109/L & $198.5 \pm 32.7$ & $217.1 \pm 56.0$ & $225.5 \pm 63.0$ & $225.0 \pm 70.4$ & $215.8 \pm 77.2$ & 372 & 0.118 & e,i,l \\
\hline $\mathrm{PaO} 2 / \mathrm{FiO} 2, \mathrm{mmHg}$ & $334.9 \pm 12.5$ & $328.2 \pm 15.9$ & $321.2 \pm 28.2$ & $285.4 \pm 43.8$ & $243.8 \pm 33.8$ & 224 & $\varangle 0.001$ & d,e,f,g,h,i,j,k,l,m \\
\hline $\begin{array}{l}\text { Length of surgery, } \\
\text { min }\end{array}$ & $293.1 \pm 30.7$ & $275.1 \pm 24.9$ & $279.2 \pm 23.5$ & $281.0 \pm 22.3$ & $284.8 \pm 18.8$ & 298 & 0.163 & a \\
\hline $\begin{array}{l}\text { Cardiopulmonary } \\
\text { bypass time, min }\end{array}$ & $164.4 \pm 16.7$ & $167.5 \pm 21.9$ & $172.8 \pm 24.3$ & $167.4 \pm 24.1$ & $183.2 \pm 23.6$ & 163.0 & 0.212 & $\mathrm{~h}$ \\
\hline $\begin{array}{l}\text { Cross-clamp time, } \\
\text { min }\end{array}$ & $87.9 \pm 10.7$ & $88.8 \pm 13.3$ & $89.4 \pm 12.6$ & $82.8 \pm 11.6$ & $95.9 \pm 17.0$ & 103 & 0.049 & g,j,m \\
\hline $\begin{array}{l}\text { Circulatory arrest, } \\
\text { min }\end{array}$ & $45.6 \pm 5.8$ & $42.2 \pm 6.9$ & $42.2 \pm 8.3$ & $41.3 \pm 6.2$ & $46.5 \pm 12.1$ & 40.6 & 0.333 & \\
\hline $\begin{array}{l}\text { Minimum } \\
\text { temperature }\end{array}$ & $25.9 \pm 0.5$ & $26.1 \pm 0.5$ & $26.0 \pm 0.5$ & $26.0 \pm 0.5$ & $26.2 \pm 0.5$ & 25.2 & 0.312 & \\
\hline HDL-C, mmol/L & $1.5(1.2,2.2)$ & $1.8(1.3,2.3)$ & $1.3(0.9,1.8)$ & $1.0(0.9,1.5)$ & $0.9(0.8,1.5)$ & 0.8 & $\varangle 0.001$ & $c, f, g, h, j$ \\
\hline LVEF,\% & $60.5 \pm 7.6$ & $57.3 \pm 6.2$ & $58.0 \pm 6.3$ & $54.7 \pm 6.2$ & $57.7 \pm 7.6$ & 58 & 0.100 & $c, g, j$ \\
\hline \multicolumn{9}{|c|}{ a:0vs1, b:0vs2, c:0vs3, d:0vs4, e:0vs5, f:1vs2, g:1vs3, h:1vs4, i:1vs5, g:2vs3, k:2vs4, l:2vs5, m:3vs4, n:3vs5, o:4vs5. } \\
\hline $\begin{array}{l}\text { METs:Metabolic Sy } \\
\text { diastolic blood pres } \\
\text { LVEF:ventricular eje }\end{array}$ & $\begin{array}{l}\text { drome;BMI: bc } \\
\text { ure;HR: heart } \\
\text { tion fraction. }\end{array}$ & $\begin{array}{l}y \text { mass index } \\
\text { e; FBG:Fasti }\end{array}$ & $\begin{array}{l}\text { AT:Hypertens } \\
\text { blood gluco }\end{array}$ & $\begin{array}{l}\text { n; T2DM: typ } \\
\text { TG: Triglyce }\end{array}$ & $\begin{array}{l}2 \text { diabetes } \\
\text { les;HDL-C: }\end{array}$ & $\begin{array}{l}\text { litus; SBP } \\
\text { h-density }\end{array}$ & $\begin{array}{l}\text { stolic b } \\
\text { sprotein }\end{array}$ & $\begin{array}{l}\text { d pressure; DBP: } \\
\text { olesterol; }\end{array}$ \\
\hline
\end{tabular}

\section{METs components and hypoxemia}

After adjustment for some potential risk factors, such as age, male sex, HR, CAD, previous thoracic surgery, and smoking, BMI quartiles (adjusted OR $=2.616,95 \% \mathrm{Cl} 1.743-3.924, \mathrm{P}<0.001$ ), HDL (adjusted OR $=0.560,95 \% \mathrm{Cl} 0.393-0.799, P<0.001$ ) and SBP (adjusted OR = $1.646,95 \% \mathrm{Cl} 1.145-2.367, P=0.007)$ remained independent factors of hypoxemia. People with T2DM had a significantly increased risk of hypoxemia compared with those with no T2DM in all groups (adjusted OR $=5.460,95 \% \mathrm{Cl} 2.211-13.484, \mathrm{P}<0.001$ ). Compared with the first 
BMI quartile, the second, third and fourth BMI quartiles had ORs of incident hypoxemia of 6.124 (95\% Cl 1.056-35.493), 6.269 (95\% Cl 1.14234.409), and 33.918 (95\% Cl 6.084-189.102), respectively, after adjusting for potential risk factors (Table 3). 
Table 3

Impact of MetS components on patients with hypoxemia.

\begin{tabular}{|c|c|c|c|c|c|}
\hline \multirow[t]{2}{*}{ Variables } & \multicolumn{2}{|c|}{ Quartiles of components } & \multicolumn{3}{|l|}{ All } \\
\hline & Range & $\mathbf{n}$ & Hypoxemia/No Hypoxemia & OR(95\%Cl) & P Value \\
\hline $\mathrm{HT}, \mathrm{n}(\%)$ & - & 145 & $32 / 113$ & $1.827(0.820-4.067)$ & 0.140 \\
\hline T2DM,n(\%) & - & 45 & $19 / 26$ & $5.460(2.211-13.484)$ & $<0.001$ \\
\hline \multirow[t]{5}{*}{$\mathrm{BMI}, \mathrm{Kg} / \mathrm{m} 2$} & Per quartile & 271 & $48 / 223$ & $2.616(1.743-3.924)$ & $<0.001$ \\
\hline & $\mathrm{Q} 1 \leq 24.44$ & 68 & $2 / 66$ & - & - \\
\hline & $24.22<\mathrm{Q} 2 \leq 26.02$ & 68 & $8 / 60$ & $6.124(1.056-35.493)$ & 0.043 \\
\hline & $26.02<\mathrm{Q} 3 \leq 27.88$ & 68 & $10 / 58$ & $6.269(1.142-34.409)$ & 0.035 \\
\hline & $27.88<\mathrm{Q} 4$ & 67 & $28 / 39$ & $33.918(6.084-189.102)$ & $<0.001$ \\
\hline \multirow[t]{5}{*}{$\mathrm{TG}, \mathrm{mmol} / \mathrm{L}$} & Per quartile & 271 & $48 / 223$ & $1.236(0.870-1.756)$ & 0.238 \\
\hline & $\mathrm{Q} 1 \leq 1.04$ & 70 & $11 / 59$ & - & - \\
\hline & $1.04<\mathrm{Q} 2 \leq 1.23$ & 66 & $9 / 57$ & $1.370(0.411-4.569)$ & 0.608 \\
\hline & $1.23<\mathrm{Q} 3 \leq 1.49$ & 67 & $5 / 62$ & $0.261(0.062-1.091)$ & 0.066 \\
\hline & $1.69<Q 4$ & 68 & $23 / 45$ & $2.268(0.696-7.384)$ & 0.174 \\
\hline \multirow[t]{5}{*}{$\mathrm{HDL}-\mathrm{C}, \mathrm{mmol} / \mathrm{L}$} & Per quartile & 271 & $48 / 223$ & $0.560(0.393-0.799)$ & 0.001 \\
\hline & $\mathrm{Q} 1 \leq 1.15$ & 65 & $23 / 42$ & - & - \\
\hline & $1.15<\mathrm{Q} 2 \leq 1.56$ & 71 & $9 / 62$ & $0.517(0.157-1.708)$ & 0.279 \\
\hline & $1.56<\mathrm{Q} 3 \leq 2.12$ & 68 & $10 / 58$ & $0.383(0.126-1.167)$ & 0.091 \\
\hline & $2.12<\mathrm{Q} 4$ & 67 & $6 / 61$ & $0.091(0.024-0.351)$ & $<0.001$ \\
\hline \multirow[t]{5}{*}{ SBP,mmHg } & Per quartile & 271 & $48 / 223$ & $1.646(1.145-2.367)$ & 0.007 \\
\hline & $\mathrm{Q} 1 \leq 135$ & 66 & $5 / 61$ & - & - \\
\hline & $135<\mathrm{Q} 2 \leq 143$ & 70 & $11 / 59$ & $4.178(0.901-19.367)$ & 0.068 \\
\hline & $143<\mathrm{Q} 3 \leq 153$ & 68 & $13 / 55$ & $3.265(0.737-14.462)$ & 0.119 \\
\hline & $153<Q 4$ & 67 & $19 / 48$ & $7.367(1.676-32.377)$ & 0.008 \\
\hline \multirow[t]{5}{*}{ DBP,mmHg } & Per quartile & 271 & $48 / 223$ & $0.988(0.678-1.441)$ & 0.952 \\
\hline & $\mathrm{Q} 1 \leq 75$ & 70 & $10 / 60$ & - & \\
\hline & $75<\mathrm{Q} 2 \leq 80$ & 69 & $10 / 59$ & $0.606(0.153-2.399)$ & 0.475 \\
\hline & $80<\mathrm{Q} 3 \leq 84$ & 62 & $16 / 46$ & $1.148(0.351-4.180)$ & 0.834 \\
\hline & $84<\mathrm{Q} 4$ & 70 & $12 / 58$ & $0.557(0.142-2.182)$ & 0.401 \\
\hline \multirow[t]{5}{*}{$\mathrm{FBG}, \mathrm{mmol} / \mathrm{L}$} & Per quartile & 271 & $48 / 223$ & $0.976(0.698-1.366)$ & 0.889 \\
\hline & $\mathrm{Q} 1 \leq 4.58$ & 66 & $12 / 54$ & - & \\
\hline & $4.62<\mathrm{Q} 2 \leq 4.93$ & 68 & $13 / 55$ & $0.989(0.312-3.141)$ & 0.986 \\
\hline & $4.93<\mathrm{Q} 3 \leq 5.29$ & 69 & $6 / 63$ & $0.353(0.082-1.522)$ & 0.163 \\
\hline & $5.29<\mathrm{Q} 4$ & 68 & $17 / 51$ & $0.951(0.303-2.987)$ & 0.932 \\
\hline \multicolumn{6}{|c|}{$\begin{array}{l}\text { OR:odds ratio; Cl:confdence interval; METs:Metabolic Syndrome; BMI: body mass index; HT:Hypertension; T2DM: type } 2 \text { diabetes mellitus; } \\
\text { HDL-C:high-density lipoprotein cholesterol, TG:Triglycerides, FBG:Fasting blood glucose; SBP: systolic blood pressure; DBP: diastolic blooc } \\
\text { pressure. }\end{array}$} \\
\hline \multicolumn{6}{|c|}{$\begin{array}{l}\text { a Multiple adjustment for Age,Male,HR,CAD,Previous thoracic surgey,Aortic valve disease,Marfan syndrome,smoker,LVEF, Length of } \\
\text { surgey,Cardiopulmonary bypass time,Cross-clamp time, Circulatory arrest,Minimum temperature,TroponinI,WBC,PLT,RBC,ICU stay } \\
\text { time,Hospital stay time,Mechanical ventilation time,PaO2/FiO2,Cr,eGFR,UA. }\end{array}$} \\
\hline
\end{tabular}




\section{METs and hypoxemia}

Table 4 shows the results of multivariate logistic regression analysis of the association between the incidence of hypoxemia and MetS. There were five models that adjusted for age, male sex, HR, CAD, previous thoracic surgery, aortic valve disease, Marfan syndrome, smoking status, LVEF, length of surgery, cardiopulmonary bypass time, cross-clamp time, circulatory arrest, minimum temperature, troponin I, WBCs, PLTs, RBCs, ICU stay time, hospital stay time, mechanical ventilation time, PaO2/FiO2, Cr, eGFR, UA, elevated body mass index, elevated blood pressure, elevated fasting glucose, reduced high-density lipoprotein cholesterol, and elevated triglycerides. The ORs were 17.112, 20.521, $31.229,40.132$, and 68.053 for MetS in Models 1, 2, 3, 4, and 5, respectively (all $P<0.05$ ).

Table 4

Odds ratio and $95 \%$ confdence interval for hypoxemia

\begin{tabular}{|c|c|c|}
\hline \multirow[t]{2}{*}{ Models } & \multicolumn{2}{|l|}{ MetS } \\
\hline & OR(95\%Cl) & P Value \\
\hline Model 1 & $17.112(7.742-37.821)$ & $<0.001$ \\
\hline Model 2 & $20.521(8.921-47.203)$ & $<0.001$ \\
\hline Model 3 & $31.229(11.295-86.341)$ & $<0.001$ \\
\hline Model 4 & 40.132(5.461-294.906) & $<0.001$ \\
\hline Model 5 & $68.053(2.026-2283.417)$ & 0.019 \\
\hline \multicolumn{3}{|c|}{ Model 1:adjusted for Age,Male,HR; } \\
\hline \multicolumn{3}{|c|}{ Model 2:adjusted for Age,Male,HR,CAD,Previous thoracic surgey,Aortic valve disease,Marfan syndrome; } \\
\hline \multicolumn{3}{|c|}{$\begin{array}{l}\text { Model 3:adjusted for Age,Male,HR,CAD,Previous thoracic surgey,Aortic valve disease,Marfan syndrome,smoker,LVEF, Length of } \\
\text { surgey,Cardiopulmonary bypass time,Cross-clamp time, Circulatory arrest,Minimum temperature; }\end{array}$} \\
\hline \multicolumn{3}{|c|}{$\begin{array}{l}\text { Model 4:adjusted for Age,Male,HR,CAD,Previous thoracic surgey,Aortic valve disease,Marfan syndrome,smoker,LVEF, Length of } \\
\text { surgey,Cardiopulmonary bypass time,Cross-clamp time, Circulatory arrest,Minimum temperature,Troponinl,WBC,PLT,RBC,ICU stay } \\
\text { time,Hospital stay time,Mechanical ventilation time,PaO2/FiO2,Cr,eGFR,UA; }\end{array}$} \\
\hline \multicolumn{3}{|c|}{$\begin{array}{l}\text { Model 5:adjusted for Age,Male,HR,CAD,Previous thoracic surgey,Aortic valve disease,Marfan syndrome,smoker,LVEF, Length of } \\
\text { surgey,Cardiopulmonary bypass time,Cross-clamp time, Circulatory arrest,Minimum temperature,TroponinI,WBC,PLT,RBC,ICU stay } \\
\text { time,Hospital stay time,Mechanical ventilation time,PaO2/FiO2,Cr,eGFR,UA,elevated body mass index, elevated blood pressure,elevated } \\
\text { fasting glucose,reduced high-density lipoprotein cholesterol,elevated triglycerides. }\end{array}$} \\
\hline
\end{tabular}

\section{METs scoring system and ROC curve analysis}

Based on the regression coefficient, a point was assigned to each METs component. Elevated BMI was given 2 points, elevated BP was given 2 points, elevated FBG was given 1 point, reduced HDL was given 1 point, and elevated TG was given 1 point (Table 5). ROC curves were constructed to evaluate the scoring system. The AUCs were $0.852(95 \% \mathrm{Cl}: 0.789-0.914)$ in all patients, $0.728(95 \% \mathrm{Cl}: 0.573-0.882)$ in patients with METs and 0.744 (95\% Cl: 0.636-0.853) in patients without METs (Table 6 Fig. 3 ).

Table 5

Multivariable analysis of the METs components.

\begin{tabular}{|llll|}
\hline Variables & OR(95\%Cl) & $P$ Value & Regression Coefficeint \\
\hline Elevated BMI & $12.084(4.193-34.828)$ & $<0.001$ & 2.482 \\
\hline Elevated BP & $9.829(0.867-111.455)$ & 0.065 & 2.285 \\
\hline Elevated FBG & $5.814(2.538-13.318)$ & $<0.001$ & 1.760 \\
\hline Reduced HDL-C & $5.300(2.358-11.914)$ & $<0.001$ & 1.668 \\
\hline Elevated TG & $2.822(1.100-7.238)$ & 0.031 & 1.037 \\
\hline BMl: body mass index; BP: blood pressure; FBG:Fasting blood glucose; HDL-C: high-density lipoprotein cholesterol; TG: Triglycerides. \\
\hline
\end{tabular}


Table 6

The ROC Curve analysis of the METs with hypoxemia.

\begin{tabular}{|lllllll|}
\hline Factors & AUC & $P$ & $95 \% \mathrm{Cl}$ & $\mathrm{Se}(\%)$ & $\mathrm{Sp}(\%)$ & Cut off point \\
\hline All Patients & 0.852 & $<0.001$ & $0.789-0.914$ & $85.40 \%$ & $70.40 \%$ & 4 \\
MetS & 0.728 & 0.012 & $0.573-0.882$ & $81.50 \%$ & $52.90 \%$ & 6 \\
\hline non-MetS & 0.744 & $<0.001$ & $0.636-0.853$ & $76.20 \%$ & $68.40 \%$ & 3 \\
\hline
\end{tabular}

\section{Discussion}

In this study, we demonstrated that METs components could significantly predict the incidence of hypoxemia in ATAAD patients according to multivariable and subgroup analyses. After adjusting for confounding factors, METs was also an independent risk factor for hypoxemia. Among METs components, BMI was the strongest predictor of hypoxemia, and the scoring system showed good predictive power.

As a result of high blood pressure, the aortic intima tears and progressive separation of the aortic wall layers results in the formation of a false lumen; this involves the ascending aorta and is classified as ATAAD [11]. With the advancement of surgery and postoperative management, the mortality of ATAAD has decreased significantly. Perioperative complications, including hepatic dysfunction, acute renal failure and neurological complications, are the main cause of death in ATAAD patients[12]. The occurrence of hypoxemia, a common complication, reaches $51 \%$ after surgery, which may lead to acute lung injury and influence recovery from the disease[13]. The main underlying mechanisms of hypoxemia after surgery in ATAAD remain unclear. Previous studies found that hypoxemia may be associated with an imbalance in ventilation and perfusion during acute bleeding [14]. In addition, inflammatory reactions and oxidative stress play an important role, damaging alveolar epithelial and capillary endothelial cells [4]. Ming Gong et al. enrolled 112 consecutive ATAAD patients who underwent surgery. They found that $\mathrm{BMI}(\mathrm{OR}=1.473)$ and female sex $(\mathrm{OR}=12.978)$ were independent risk factors for hypoxemia after multivariate logistic regression analysis [15]. Recently, a cohort study with 172 ATAAD patients explored inflammation biomarkers, such as interleukin-6 and C-reactive protein, associated with the incidence of preoperative hypoxemia [5].

METs comprises five components, BMI, blood pressure, fasting plasma glucose, high-density lipoprotein and triglycerides, which are often ignored in clinic practice [16]. It also represents a cluster of metabolic abnormalities that reflect changes in human physical performance, such as insulin resistance and neurohormonal activation. In the final common pathway, a series of inflammation signaling cascades are triggered, leading to clinical manifestations[17]. We found that METs was robustly associated with hypoxemia in logistic and subgroup analyses, especially with respect to BMI. The present study provides new insight for clinical practice in that METs may indicate an inflammatory state in the body and should be given more attention. It also indicates that the pathogenesis of hypoxemia may be a multifactorial process. Inflammation, insulin resistance and lipid abnormalities exert synergistic antitumor effects on the process of hypoxemia.

Several studies have found that BMI is an independent risk factor for hypoxemia [5-6]. Abundant amounts of cytokines and reactive oxygen species are released from adipose tissue in obesity, which leads to abnormal ventilation perfusion and decreased pulmonary gas exchange [18]. Previous studies have shown that hypoxemia is associated with decreased insulin sensitivity and varying degrees of insulin resistance [19]. At high blood glucose levels, oxygen transport and carbon monoxide diffusing capacity are decreased in the lungs [20]. In ATAAD patients, the incidence of T2DM was higher in the severe hypoxemia group than in the nonsevere hypoxemia group $(12.1 \% v s .1 .4 \%, P=0.05)$ [15]. There is still some controversy about the relationship between blood pressure and hypoxemia. Guo $Z$ et al. found that systolic blood pressure was a protective factor against preoperative hypoxemia in ATAAD patients [4]. However, systolic blood pressure was higher in the hypoxemia group. Blood pressure is reflective of the sympathetic state and systemic vascular resistance [21]. When the renin-angiotensin system induces inflammatory cascades, alveolar capillary membrane permeability and pulmonary vascular resistance are increased, leading to hypoxemia after surgery [22]. Lipids, an important factor in METs, play an important role in the modulation of inflammation. In addition, the hypoxia-inducible factor 1-vascular endothelial growth factor pathway is important in hypoxia and is regulated by high-density lipoproteins [23]. Triglyceride levels are also associated with hypoxia-inducible lipid droplet-associated protein and hypoxia inducible gene-2, which involve the process of hypoxemia [24]. In our study, reduced HDL-C and elevated TG levels were independent risk factors for hypoxemia. In the future, large studies need to be conducted to confirm the role of high-density lipoproteins and triglycerides in the development of hypoxemia.

To date, several METs diagnostic criteria, including the National Cholesterol Education Program (NCEP), International Diabetes Federation (IDF), and American Heart Association/National Heart, Lung, and Blood Institute (AHA/NHLBI) criteria, have been proposed. Considering cardiovascular disease, the NCEP METs definition may be more suitable in the Chinese population. Compared to the AHA/NHLBI and IDF criteria, the NCEP criteria better detect the prevalence of cardiovascular disease (OR: 1.40) [25]. Therefore, we chose the NCEP criteria seemed to be more suitable for our study.

Page $10 / 15$ 


\section{Limitations}

Our study has some limitations. First, this was an observational study with a single center and a small number of enrolled patients, which may have introduced selection bias. Second, the underlying mechanistic link between MetS and hypoxemia is not clear, and unidentified risk factors may affect the incidence of hypoxemia. Further large and randomized controlled trials need to be performed to confirm our results. Furthermore, in patients undergoing surgery, the experience of the individual surgeon may have some influence.

\section{Conclusions}

For ATAAD patients, the occurrence of hypoxemia after surgery seems tightly linked to METs, especially BMI. After adjusting for potential risk factors and establishing a scoring system, METs was an independent risk factor for hypoxemia. Our research indicates that hypoxemia may be a multifactorial process and that endocrine disorders that activate systemic inflammation may play an important role in ATAAD patients after surgery.

\section{Declarations}

\section{Ethics approval and consent to participate}

The study protocol was approved by the Institutional Review Board of The First Hospital of Hebei Medical University, Shijiazhuang Hebei, China.

\section{Consent for publication}

Yes

\section{Availability of data and materials}

Data available on request from the authors

\section{Competing interests}

The authors declare that they have no competing interests

\section{Funding}

None

\section{Authors' contributions}

Like Zhang: designed the work and wrote manuscript;

Lei Zhang: acquisition and analysis;

Zengren Zhao: substantively revised manuscript;

Yun Liu: obtain data;

Juzeng Wang: obtain data;

Mengye Niu:analysis data;

Xiansheng Zhao:analysis data.

\section{Acknowledgements}

None

\section{References}

1. Zhu Y, Lingala B, Baiocchi M,et al. Type A Aortic Dissection-Experience Over 5 Decades: JACC Historical Breakthroughs in Perspective. J Am Coll Cardiol. 2020;76:1703-1713. 
2. Kang DH, Kim JW, Kim SH,et al. Management of acute type A aortic dissection with acute lower extremities malperfusion. J Cardiothorac Surg. 2019;14: 206.

3. Ge H, Jiang Y, Jin Q,et al. Nomogram for the prediction of postoperative hypoxemia in patients with acute aortic dissection.BMC Anesthesiol. 2018; 18: 146.

4. Guo Z, Yang Y, Zhao M, et al.Preoperative hypoxemia in patients with type A acute aortic dissection: a retrospective study on incidence, related factors and clinical significance.J Thorac Dis. 2019;11: 5390-5397.

5. Duan XZ, Xu ZY, Lu FL,et al. Inflammation is related to preoperative hypoxemia in patients with acute Stanford type A aortic dissection.J Thorac Dis. 2018;10: 1628-1634.

6. Wu Z, Wang Z, Wu H, et al. Obesity is a risk factor for preoperative hypoxemia in Stanford A acute aortic dissection. Medicine (Baltimore). 2020;99: e19186.

7. Ota H, Fujita Y, Yamauchi M,et al.Relationship Between Intermittent Hypoxia and Type 2 Diabetes in Sleep Apnea Syndrome.Int J Mol Sci. 2019;20(19):4756.

8. Yang R, Sikka G, Larson J,et al.Restoring leptin signaling reduces hyperlipidemia and improves vascular stiffness induced by chronic intermittent hypoxia.Am J Physiol Heart Circ Physiol. 2011,300: H1467-76.

9. Torres S, Fabersani E, Marquez A, et al. Adipose tissue inflammation and metabolic syndrome. The proactive role of probiotics.Eur J Nutr. 2019;58:27-43.

10. Ebrahimi $\mathrm{H}$, Emamian $\mathrm{MH}$, et al. Comparison of the accuracy of three diagnostic criteria and estimating the prevalence of metabolic syndrome: A latent class analysis. J Res Med Sci. 2019; 24: 108.

11. Koechlin L, Schuerpf J, Bremerich J,et al. Acute aortic dissection with entry tear at the aortic arch: Iong-term outcome.Interact Cardiovasc Thorac Surg. 2021;32: 89-96.

12. Wang TKM, Wei D, Evans T,et al. Surgery for Type A Aortic Dissection: 14-Year Contemporary Cohort Study.Heart Lung Circ. 2020;29(8):1210-1216.

13. Ma GG, Hao GW, Lai H,et al. Initial clinical impact of inhaled nitric oxide therapy for refractory hypoxemia following type A acute aortic dissection surgery.J Thorac Dis.2019;11:495-504.

14. Naito R, Sakakura K, Kasai T,et al. Aortic dissection is associated with intermittent hypoxia and re-oxygenation. Heart Vessels.2012;27:265-70.

15. Gong M, Wu Z, Xu S,et al.Increased risk for the development of postoperative severe hypoxemia in obese women with acute type a aortic dissection. J Cardiothorac Surg.2019;14:81.

16. Varghese JF, Patel R, Yadav UCS.Novel Insights in the Metabolic Syndrome-induced Oxidative Stress and Inflammation-mediated Atherosclerosis.Curr Cardiol Rev.2018;14:4-14.

17. Garrido-Torres N, Rocha-Gonzalez I, Alameda L,et al. Metabolic syndrome in antipsychotic-naive patients with first-episode psychosis: a systematic review and meta-analysis.Psychol Med. 2021:1-14.

18. Zhou J, Pan J, Yu Y,et al. Independent risk factors of hypoxemia in patients after surgery with acute type A aortic dissection.Ann Palliat Med. 2021;10:7388-7397.

19. Newhouse LP, Joyner MJ, Curry TB,et al.Three hours of intermittent hypoxia increases circulating glucose levels in healthy adults.Physiol Rep. 2017;5:e13106.

20. Duennwald T, Gatterer H, Groop PH,et al.Effects of a single bout of interval hypoxia on cardiorespiratory control and blood glucose in patients with type 2 diabetes.Diabetes Care. 2013; 36: 2183-9.

21. Bilo G, Caravita S, Torlasco C,et al. Blood pressure at high altitude: physiology and clinical implications.Kardiol Pol.2019;77:596-603.

22. Garcia VP, Rocha HNM, Rocha MP, et al.Hypertension impairs hypoxia-induced angiogenesis in men.J Hypertens. 2020;38:1131-1139.

23. Tan JT, Prosser HC, Vanags LZ, et al.High-density lipoproteins augment hypoxia-induced angiogenesis via regulation of post-translational modulation of hypoxia-inducible factor 1a.FASEB J. 2014;28:206-17.

24. Padmanabha Das KM, Wechselberger L, Liziczai M,et al.Hypoxia-inducible lipid droplet-associated protein inhibits adipose triglyceride lipase.J Lipid Res 2018 03;59:531-541.

25. Li W, Song F, Wang X, et al. Relationship between metabolic syndrome and its components and cardiovascular disease in middle-aged and elderly Chinese population: a national cross-sectional survey. BMJ Open. 2019;9:e027545.

\section{Figures}




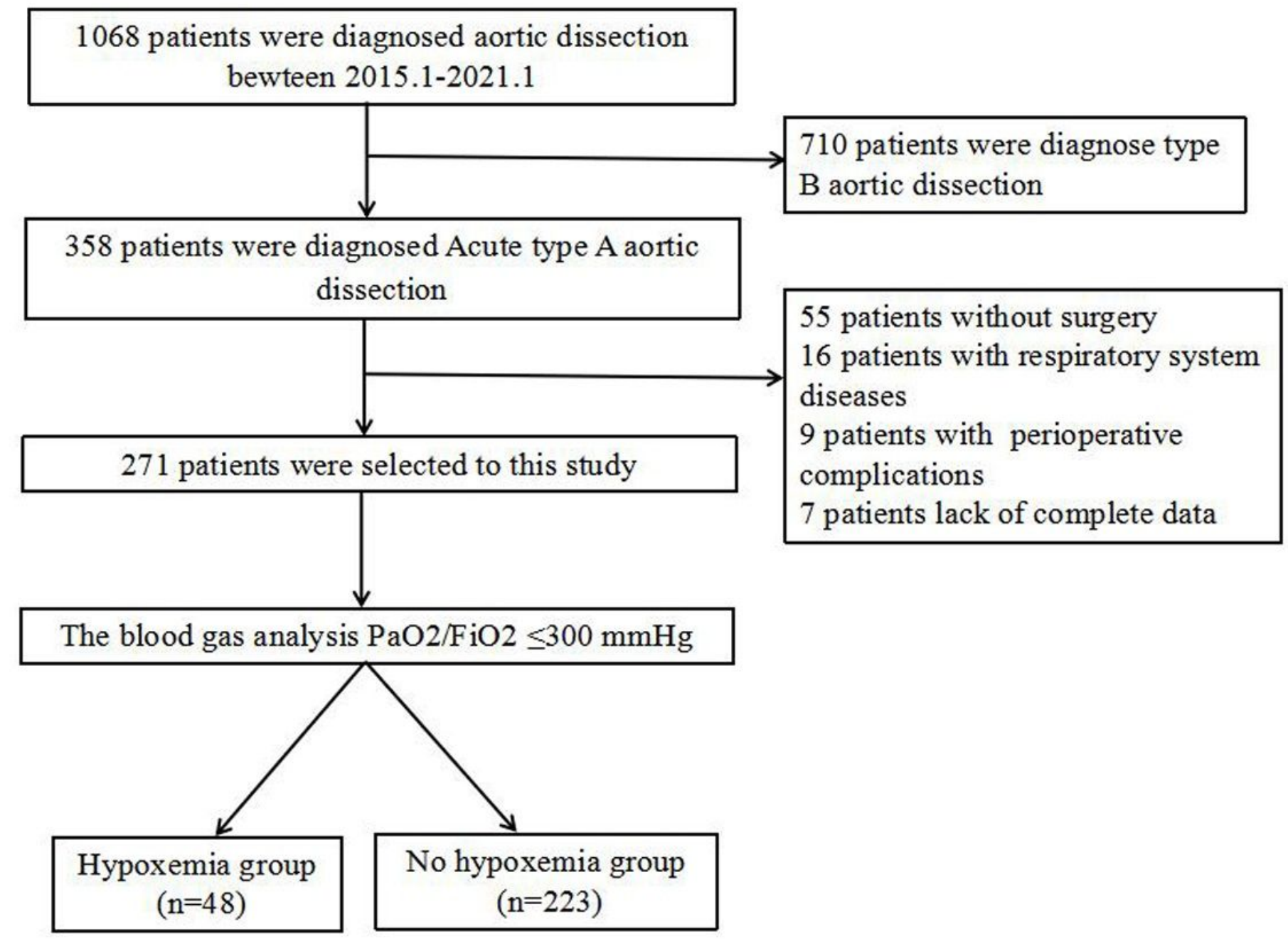

Figure 1

Population flowchart of enrolled patients. 


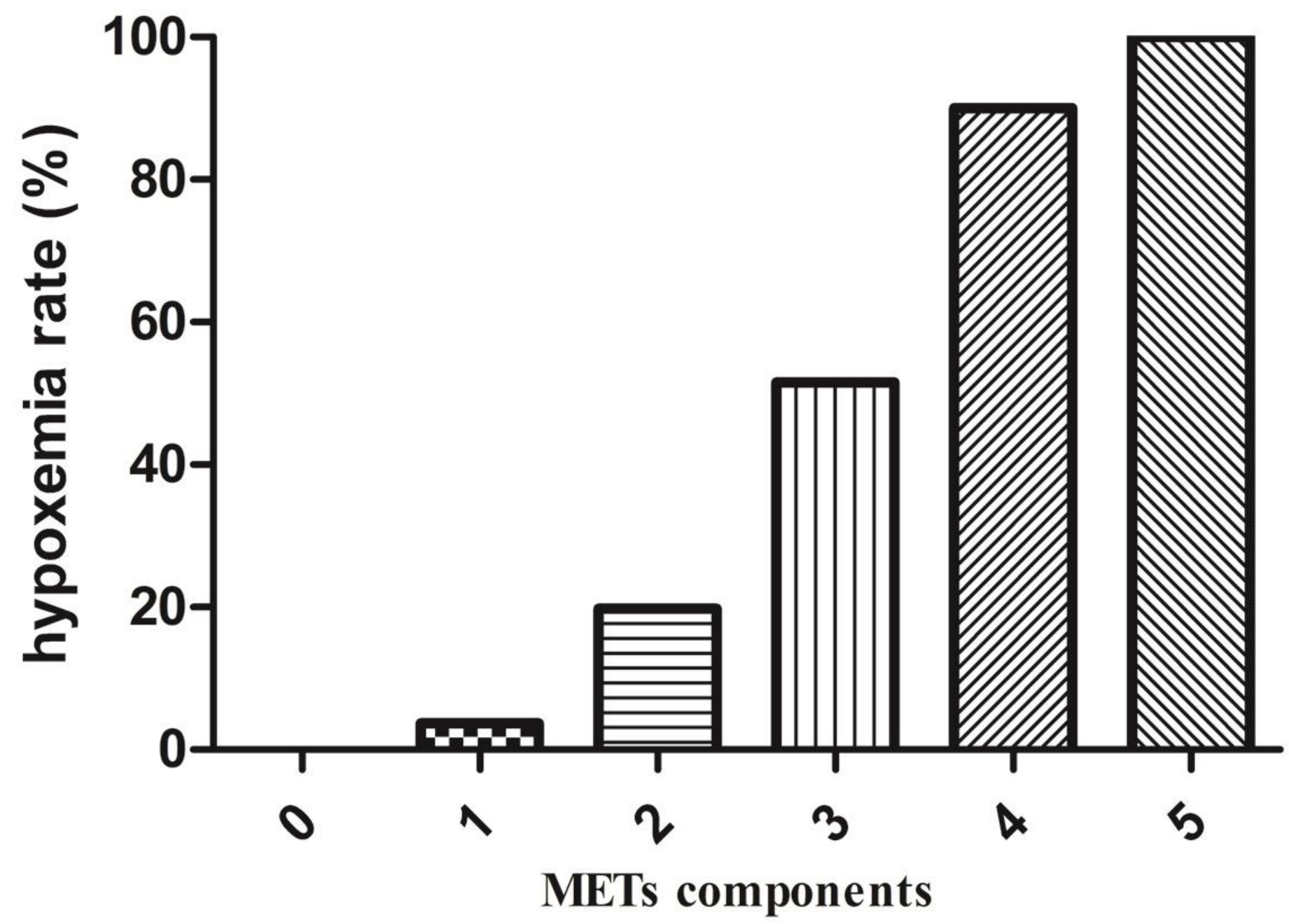

Figure 2

Hypoxemia rate of metabolic syndrome components. 


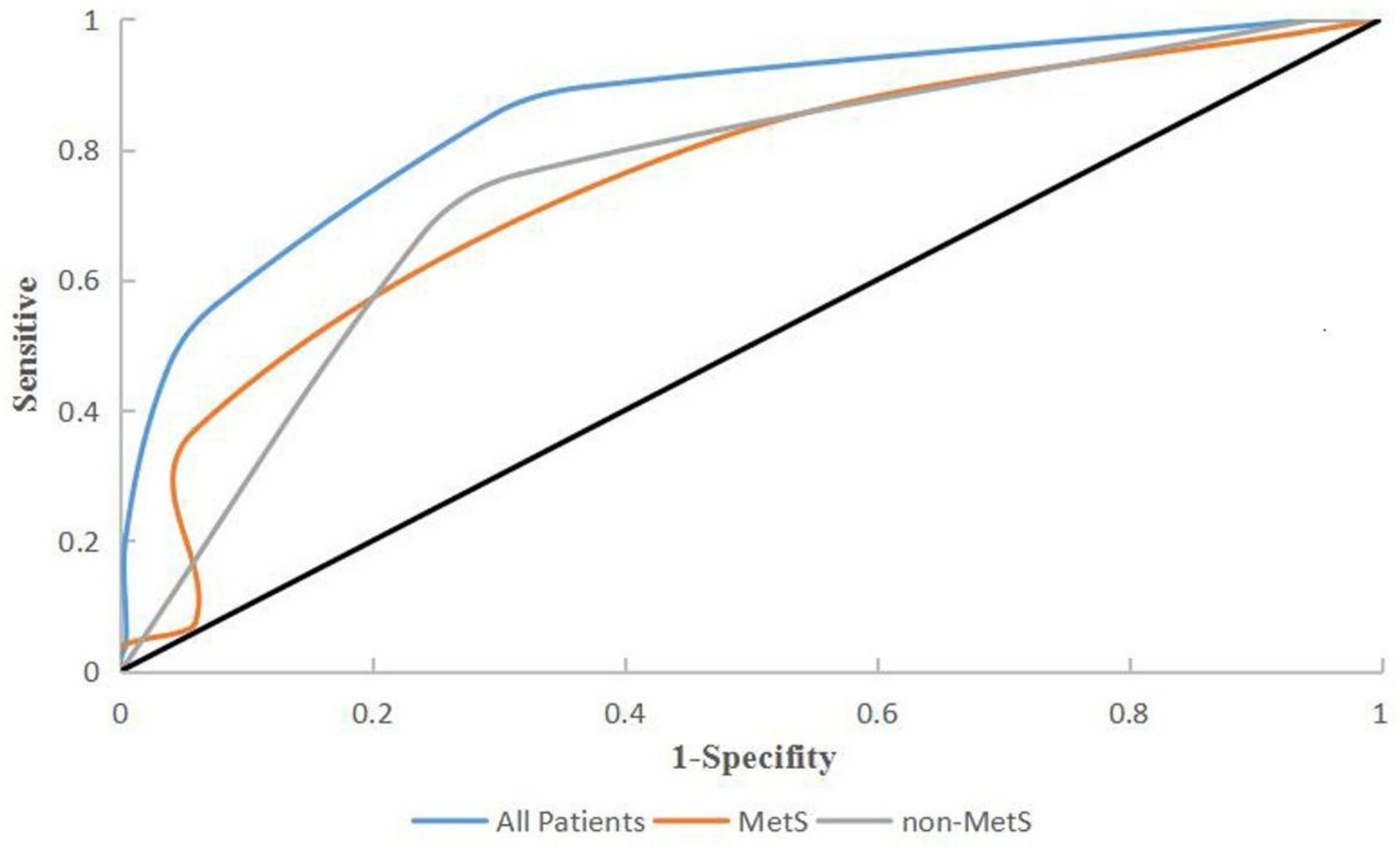

Figure 3

ROC curve analysis of the scoring system for the prediction of Hypoxemia. 